Pacific

Journal of

Mathematics

COMPLEXES OF INJECTIVE WORDS AND THEIR COMMUTATION CLASSES

JAKOB JONSSON AND VOLKMAR WELKER

Volume $243 \quad$ No. 2

December 2009 


\title{
COMPLEXES OF INJECTIVE WORDS AND THEIR COMMUTATION CLASSES
}

\author{
JAKOB JONSSON AND VOLKMAR WELKER
}

\begin{abstract}
Let $S$ be a finite alphabet. An injective word over $S$ is a word over $S$ such that each letter in $S$ appears at most once in the word. For an abstract simplicial complex $\Delta$, let $\Gamma(\Delta)$ be the Boolean cell complex whose cells are indexed by all injective words over the sets forming the faces of $\Delta$. The boundary of a cell indexed by a given word $w$ consists of those cells that are indexed by subwords of $w$.

For a partial order $P$ on $S$, we study the subcomplex $\Gamma(\Delta, P)$ of $\Gamma(\Delta)$ consisting of those cells that are indexed by words whose letters are arranged in increasing order with respect to some linear extension of the order $P$.

For a graph $G=(S, E)$ on vertex set $S$ and a word $w$ over $S$, let [w] be the class of all words that we can obtain from $w$ via a sequence of commutations $s s^{\prime} \rightarrow s^{\prime} s$ such that $\left\{s, s^{\prime}\right\}$ is not an edge in $E$. We study the Boolean cell complex $\Gamma / G(\Delta)$ whose cells are indexed by commutation classes $[w]$ of words indexing cells in $\Gamma(\Delta)$. We prove:

- If $\Delta$ is shellable then so are $\Gamma(\Delta, P)$ and $\Gamma / G(\Delta)$.

- If $\Delta$ is Cohen-Macaulay (respectively sequentially Cohen-Macaulay) then so are $\Gamma(\Delta, P)$ and $\Gamma / G(\Delta)$.

- The complex $\Gamma(\Delta)$ is partitionable.
\end{abstract}

Our work generalizes work by Farmer and by Björner and Wachs on the complex of all injective words.

\section{Introduction}

A word $\omega$ over a finite alphabet $S$ is called injective if no letter appears more than once; that is $\omega=\omega_{1} \cdots \omega_{r}$ for some $\omega_{1}, \ldots, \omega_{r} \in S$ and $\omega_{i} \neq \omega_{j}$ for $1 \leq i<j \leq r$. For $n+1=\# S$ we denote by $\Gamma_{n}$ the set of all injective words on $S$. A word

MSC2000: primary 05E99, 52B22; secondary 18F25.

Keywords: injective word, Boolean cell complex, simplicial complex, Cohen-Macaulay complex, shellable complex.

First author supported by Graduiertenkolleg "Combinatorics, Geometry, Computation”, DFG-GRK 588/2. Both authors were supported by EU Research Training Network "Algebraic Combinatorics in Europe", grant HPRN-CT-2001-00272. 
$\omega=\omega_{1} \cdots \omega_{r}$ with $r$ letters is said to be of length $r$. A subword of a word $\omega_{1} \cdots \omega_{r}$ is a word $\omega_{j_{1}} \cdots \omega_{j_{s}}$ such that $1 \leq j_{1}<\cdots<j_{s} \leq r$. Clearly, a subword of an injective word is injective. We order $\Gamma_{n}$ by saying that $\rho_{1} \cdots \rho_{s} \preceq \omega_{1} \cdots \omega_{r}$ if and only if $\rho_{1} \cdots \rho_{s}$ is a subword of $\omega_{1} \cdots \omega_{r}$. We write $c(w)$ for the content $\left\{\omega_{1}, \ldots, \omega_{r}\right\}$ of the word $w=\omega_{1} \cdots \omega_{r}$. Then for any $A \subseteq c(w)$ there is a unique subword $v \preceq w$ of $w$ with $A=c(v)$. This implies the well-known fact (see [Farmer 1979]) that $\Gamma_{n}$ together with the partial order $\preceq$ is the face poset of a Boolean cell complex. Recall that a Boolean cell complex is a regular CW-complex for which the poset of faces of each cell is a Boolean lattice. Clearly, simplicial complexes are special cases of Boolean cell complexes. From now on we will identify the poset $\Gamma_{n}$ with the Boolean cell complex with face poset $\Gamma_{n}$. In particular, we also identify the injective words of length $d+1$ with $d$-cells. Thus the faces of a given $d$-cell $w$ are the cells corresponding to all subwords of $w$.

The complex $\Gamma_{n}$ is a well-studied object. Farmer [1979] demonstrated that $\Gamma_{n}$ is homotopy equivalent to a wedge of spheres of top dimension. Björner and Wachs [1983] proved the stronger result that $\Gamma_{n}$ is shellable. See [Hanlon and Hersh 2004; Reiner and Webb 2004] for further refinements. Our generalizations are partly motivated by specific examples of complexes of injective words that are used in algebraic $K$-theory; see for example [van der Kallen 1980; Suslin 1984; Gerdes 1991; Knudson 2001; Mirzaii and van der Kallen 2002; Kerz 2005]. After the next paragraph, when the main objects of the paper are defined, we will make this connection more precise.

All our simplicial complexes and Boolean cell complexes are assumed to be finite. In this paper, we generalize $\Gamma_{n}$ in three directions:

- Given a simplicial complex $\Delta$ whose ground set is the alphabet $S$, we define a subcomplex $\Gamma(\Delta)$ of $\Gamma_{n}$ by restricting to injective words $w \in \Gamma_{n}$ such that the content $c(w)$ is a face of $\Delta$.

- Given a partially ordered set $P=\left(S, \leq_{P}\right)$ on the alphabet $S$, we define a subcomplex of $\Gamma_{n}$ by restricting to words $\omega_{1} \cdots \omega_{r}$ such that $i<j$ whenever $\omega_{i}<_{P} \omega_{j}$. For a simplicial complex $\Delta$ on $S$ we write $\Gamma(\Delta, P)$ for the set of all words $w \in \Gamma(\Delta)$ satisfying this restriction. In particular, $\Gamma(\Delta, P) \cong \Delta$ if $P$ is a total order and $\Gamma(\Delta, P)=\Gamma(\Delta)$ if $P$ is an antichain.

- Given a graph $G=(S, E)$ on the alphabet $S$, we define the equivalence class [w] of an injective word $w \in \Gamma_{n}$ as the set of all words $v$ that can be obtained from $w$ by applying a sequence of commutations $s s^{\prime} \rightarrow s^{\prime} s$ such that $\left\{s, s^{\prime}\right\}$ is not an edge in $E$. For a simplicial complex $\Delta$ over $S$ we write $\Gamma / G(\Delta)$ for the set of equivalence classes $[w]$ of injective words $w$ with content $c(w)$ in $\Delta$. We order $\Gamma / G(\Delta)$ by saying $[v] \preceq[w]$ if there are representatives $v^{\prime} \in[v]$ and $w^{\prime} \in[w]$ such that $v^{\prime} \preceq w^{\prime}$. In particular, if $E=\varnothing$ then $\Gamma / G(\Delta) \cong \Gamma(\Delta)$. 
Before we proceed we list a few other appearances of complexes $\Gamma(\Delta)$. In connection with work on Grassmann homology [Suslin 1984; Gerdes 1991], for a finite dimensional vector space $V$ and a number $s \leq \operatorname{dim} V$, the complex $\Gamma\left(\Delta_{V, s}\right)$ appears for the simplicial complex $\Delta_{V, s}$ of all collections $\left\{v_{0}, \ldots, v_{l}\right\}$ of vectors from $V$ such that any subset of size at most $s$ is linearly independent. It is crucial that homology vanishes except for the top degree (see [Suslin 1984, Lemma 2.2] for the case $s=\operatorname{dim} V$ ). In [Kerz 2005] the same vanishing of the homology of the "classical" complex $\Gamma_{n}$ is applied. Finally, in [Mirzaii and van der Kallen 2002] several classes of Boolean cell complexes are studied. For example, for a given ring $R$ the complex $\Gamma\left(\Delta_{R}\right)$ is studied for the simplicial complex $\Delta_{R}$ of all subsets $\left\{x_{0}, \ldots, x_{r}\right\}$ of $R$ such that the ideal generated by $x_{0}, \ldots, x_{n}$ is $R$. Again vanishing of homology of this complex in low dimensions is applied in algebraic $K$-theory. All these examples have in common that they emerge in the following way. Given a group $G$ one searches for a (chain) complex with free action of $G$. This is achieved by considering the cellular chain complex of $\Gamma(\Delta)$ for a $G$-invariant simplicial complex over a ground set with free $G$-action. Recently and outside of algebraic $K$-theory, the complex $\Gamma / G(\Delta)$ has appeared in connection with Coxeter groups in [Ragnarsson and Tenner 2009]. Indeed, the topological consequence of Theorem 3.4 in that reference can be seen as a consequence of our Theorem 1.2.

After having outlined some of the motivation for the study of the objects defined above, we proceed with the development of the results. It is easy to see that $\Gamma(\Delta)$ and $\Gamma(\Delta, P)$ are lower order ideals in $\Gamma_{n}$ and therefore can be seen as subcomplexes of $\Gamma_{n}$. This in turn implies that we can also regard them as Boolean cell complexes. Slightly more care is needed to recognize $\Gamma / G(\Delta)$ as a Boolean cell complex.

\section{Lemma 1.1. $\Gamma / G(\Delta)$ is a Boolean cell complex.}

Proof. Clearly, if two words are in the same equivalence class, then their contents must coincide. Also $[v] \preceq[w]$ implies that the content of $v$ is a subset of the content of $w$. These facts show that for a word $w$ of length $r$ there is a surjective poset map from $\{[v] \mid[v] \preceq[w]\}$ to the Boolean lattice of subsets of an $r$-element set. In order to show that it is an isomorphism we need to see that if $v_{1}$ and $v_{2}$ are words with the same content and $\left[v_{1}\right],\left[v_{2}\right] \preceq[w]$ then $\left[v_{1}\right]=\left[v_{2}\right]$. We may assume that $v_{1} \preceq w$ and $v_{2} \preceq w^{\prime}$ for some $w^{\prime} \in[w]$. Since $w$ and $w^{\prime}$ are equivalent, there is a sequence of commutations that leads from $w$ to $w^{\prime}$. The commutations that involve only letters from $c\left(v_{1}\right)$ can then be used to move from $v_{1}$ to $v_{2}$. In particular, $\left[v_{1}\right]=\left[v_{2}\right]$.

The following three theorems are our main results. Their proofs are provided in the subsequent sections. The concepts from topological combinatorics used to formulate the theorems are introduced in the corresponding section. For further 
reference we refer to the survey [Björner 1995] and for particular information about sequential Cohen-Macaulay (CM) complexes to [Björner et al. 2009].

Theorem 1.2. Let $\Delta$ be a shellable simplicial complex on the vertex set $S$.

(i) Let $P=\left(S, \leq_{P}\right)$ be a partial order on $S$. Then the Boolean cell complex $\Gamma(\Delta, P)$ is shellable.

(ii) Let $G=(S, E)$ be a simple graph on $S$. Then the Boolean cell complex $\Gamma / G(\Delta)$ is shellable.

Using the preceding theorem for $\Delta$ being a simplex and poset fiber theorems from [Björner et al. 2005], we derive our second main result.

Theorem 1.3. Let $\Delta$ be a sequentially homotopy $C M$ (respectively sequentially $C M$ over $\mathbb{K}$ ) simplicial complex on the vertex set $S$.

(i) If $P=\left(S, \leq_{P}\right)$ is a partial order on $S$, then the Boolean cell complex $\Gamma(\Delta, P)$ is sequentially homotopy $C M$ (respectively sequentially $C M$ over $\mathbb{K}$ ). In particular, if $\Delta$ is homotopy $C M$ (respectively $C M$ over $\mathbb{K})$, then so is $\Gamma(\Delta, P)$.

(ii) If $G=(S, E)$ is a graph on vertex set $S$, then the Boolean cell complex $\Gamma / G(\Delta)$ is sequentially homotopy $C M$ (respectively sequentially $C M$ over $\mathbb{K}$ ). In particular, if $\Delta$ is homotopy $C M$ (respectively $C M$ over $\mathbb{K}$ ), then so is $\Gamma / G(\Delta)$.

Our third result exhibits a general property of the complexes $\Gamma(\Delta, P)$ in the case that $P$ is the antichain.

Theorem 1.4. Let $\Delta$ be a simplicial complex on vertex set $S$. Then the complex $\Gamma(\Delta)$ of injective words derived from $\Delta$ is partitionable.

\section{Auxiliary lemmas}

In this section we list some lemmas that give more insight into the structure of the complexes $\Gamma(\Delta, P)$ and $\Gamma / G(\Delta)$ and also serve as ingredients for the proofs in later sections.

For a partial order $P=\left(S, \leq_{P}\right)$ and a set $B \subseteq S$, let $\left.P\right|_{B}$ be the induced partial order on $B$. For a linear extension $w=a_{1} a_{2} \cdots a_{r}$ of $\left.P\right|_{\left\{a_{1}, a_{2}, \ldots, a_{r}\right\}}, P+w$ denotes the partial order obtained from $P$ by adding the relations $a_{i}<a_{i+1}$ for $1 \leq i \leq r-1$ and taking the transitive closure of the resulting set of relations. For example, if $a, b$ are incomparable in $P$ then $P+a b$ is the partial order obtained from $P$ by adding the relation $c<d$ for all pairs $(c, d)$ satisfying $c \leq_{P} a$ and $b \leq_{P} d$.

For a Boolean cell complex $\Gamma$ and a face $w \in \Gamma$, let $\operatorname{fdel}_{\Gamma}(w)$ denote the complex obtained by removing the face $w$ and all faces containing $w$. Let $\operatorname{st}_{\Gamma}(w)$ be the 
complex consisting of all faces $w^{\prime}$ of $\Gamma$ such that some face of $\Gamma$ contains both $w$ and $w^{\prime}$. We call $\operatorname{fdel}_{\Gamma}(w)$ the deletion of $w$ in $\Gamma$ and $\operatorname{st}_{\Gamma}(w)$ the star of $w$ in $\Gamma$.

If $\Delta$ and $\Gamma$ are simplicial complexes on disjoint ground sets, then the join $\Delta * \Gamma$ is the simplicial complex

$$
\Delta * \Gamma:=\{\sigma \cup \tau: \sigma \in \Delta, \tau \in \Gamma\} .
$$

Still restricting to simplicial complexes, we define the link of $\tau$ in $\Gamma$ to be the simplicial complex

$$
\mathrm{lk}_{\Gamma}(\tau):=\{\sigma: \sigma \cup \tau \in \Gamma, \sigma \cap \tau=\varnothing\} .
$$

Clearly, for a simplicial complex $\Gamma$ we have that $\operatorname{st}_{\Gamma}(\tau)=2^{\tau} * \mathrm{k}_{\Gamma}(\tau)$.

Lemma 2.1. Let $P=\left(S, \leq_{P}\right)$ be a partial order on $S$ and let $\sigma$ and $\tau$ be subsets of $S$. If $w$ is a linear extension of $\left.P\right|_{\sigma}$, then there exists a linear extension $w^{\prime}$ of $\left.P\right|_{\sigma \cup \tau}$ containing $w$ as a subword.

Proof. By a simple induction argument, it suffices to consider the case $\tau=\{x\}$, where $x \in S \backslash \sigma$. If $x$ is maximal in $\left.P\right|_{\tau+x}$, then we may choose $x$ to be the maximal element in $w^{\prime}$. Otherwise, let $y$ be the leftmost element in $w$ such that $x<_{P} y$. Let $w^{\prime}$ be the word obtained from $w$ by inserting $x$ just before $y$. Then $w^{\prime}$ is a linear extension of $\left.P\right|_{\sigma+x}$. Namely, if $z<_{P} x$, then $z<_{P} y$, which implies that $z$ appears before $y$ and hence before $x$ in $w^{\prime}$.

Lemma 2.2. Let $\Delta$ be a simplicial complex on the vertex set $S$ and let $P=\left(S, \leq_{P}\right)$ be a partial order on $S$. If $w \in \Gamma(\Delta, P)$, then

$$
\Gamma\left(\mathrm{st}_{\Delta}(c(w)), P+w\right)=\mathrm{st}_{\Gamma(\Delta, P)}(w)
$$

Proof. A cell $w^{\prime}$ belongs to the left-hand side if and only if $c(w) \cup c\left(w^{\prime}\right) \in \Delta$ and $w^{\prime}$ is a linear extension of $\left.(P+w)\right|_{c\left(w^{\prime}\right)}$. By Lemma 2.1, there is then a face $w^{\prime \prime}$ of $\Gamma(\Delta, P+w)$ containing $w^{\prime}$ such that $c\left(w^{\prime \prime}\right)=c(w) \cup c\left(w^{\prime}\right)$. This implies that $w^{\prime} \in \operatorname{st}_{\Gamma(\Delta, P+w)}(w)$. As a consequence,

$$
\Gamma\left(\operatorname{st}_{\Delta}(c(w)), P+w\right) \subseteq \mathrm{st}_{\Gamma(\Delta, P+w)}(w)=\operatorname{st}_{\Gamma(\Delta, P)}(w) .
$$

Conversely, $w^{\prime}$ belongs to the right-hand side if and only if there is a face $w^{\prime \prime}$ of $\Gamma(\Delta, P)$ with content $c(w) \cup c\left(w^{\prime}\right)$ such that $w^{\prime \prime}$ is a linear extension of the partial order $\left.(P+w)\right|_{c(w) \cup c\left(w^{\prime}\right)}$. This implies that the two families are identical.

\section{Shellable complexes}

A pure Boolean cell complex is a Boolean cell complex in which all cells that are maximal with respect to inclusion have the same dimension. We define the class of shellable Boolean cell complexes as follows. A finite Boolean cell complex $\Gamma$ is called shellable if $\Gamma$ satisfies one of the following two conditions: 
(i) $\Gamma=2^{\Omega}$ for some finite set $\Omega$.

(ii) $\Gamma$ is pure and there is a cell $\sigma$ in $\Gamma$ that is contained in a unique maximal cell $\tau$ such that $\operatorname{fdel}_{\Gamma}(\sigma)$ is shellable.

Note that we allow $\Omega=\varnothing$ and $\Gamma=\{\varnothing\}$ in (i) and $\sigma=\tau$ in (ii). Also in the situation of (ii) we have $\operatorname{fdel}_{\Gamma}(\sigma)=\Gamma \backslash[\sigma, \tau]$, where $[\sigma, \tau]=\{\rho: \sigma \subseteq \rho \subseteq \tau\}$.

A simple inductive argument yields the following.

Proposition 3.1. Let $\Gamma$ be a pure d-dimensional Boolean cell complex and let $\tau_{1}, \ldots, \tau_{r}$ be its inclusion maximal cells. Then $\Gamma$ is shellable if and only if there is an ordered partition of $\Gamma$ into intervals $\left[\sigma_{1}, \tau_{1}\right], \ldots,\left[\sigma_{r}, \tau_{r}\right]$ such that for $1 \leq k \leq$ $r-1$ the union $\bigcup_{i=1}^{k}\left[\sigma_{i}, \tau_{i}\right]$ is a shellable Boolean cell complex of dimension $d$. In particular, $\Gamma$ is homotopy equivalent to a wedge of $d$-spheres. The number of spheres is given by $\#\left\{j \mid \sigma_{j}=\tau_{j}\right\}$.

To sketch a proof of the proposition, suppose that $\Gamma$ satisfies (ii) above, and assume inductively that $\operatorname{fdel}_{\Gamma}(\sigma)$ admits an ordered partition into intervals $\left[\sigma_{1}, \tau_{1}\right], \ldots$, $\left[\sigma_{r}, \tau_{r}\right]$ with properties as in the proposition. Adding the interval $[\sigma, \tau]$ at the end of the partition, we obtain an ordered partition of $\Gamma$ with desired properties.

We refer to such an ordered partition as described in Proposition 3.1 as a shelling order. Indeed the usual definition of a shellable Boolean cell complex $\Gamma$ of dimension $d$ postulates that $\Gamma$ is pure and that there is linear order $\tau_{1}, \ldots, \tau_{r}$ of its $d$ dimensional cells such that the intersection of the complex generated by $\tau_{1}, \ldots, \tau_{i}$ and the cell $\tau_{i+1}$ is shellable of dimension $d-1$ for all $1 \leq i \leq r-1$. It is easy to check that the existence of such an ordering is equivalent to the existence of a shelling order in our sense.

Lemma 3.2. If $\Gamma$ is a pure Boolean cell complex and $\rho$ is a face such that $\Gamma^{1}=$ $\operatorname{fdel}_{\Gamma}(\rho)$ and $\Gamma^{2}=\operatorname{st}_{\Gamma}(\rho)$ are shellable of dimension $d$, then $\Gamma$ is shellable of dimension $d$.

Proof. Let

$$
\left[\alpha_{i 1}, \beta_{i 1}\right], \ldots,\left[\alpha_{i r_{i}}, \beta_{i r_{i}}\right]
$$

be a shelling order of $\Gamma^{i}$ for $i=1,2$. Note that each $\beta_{2 j}$ contains the face $\rho$, because each maximal face of a star complex $\operatorname{st}_{\Gamma}(\rho)$ contains $\rho$. In the interval $\left[\alpha_{2 j}, \beta_{2 j}\right]$, there is a unique minimal face $\gamma_{2 j}$ containing $\rho$. Namely, the intersection of two such faces would again lie in the interval and contain $\rho$. We claim that the above shelling order on $\Gamma^{1}$, together with

$$
\left[\gamma_{21}, \beta_{21}\right], \ldots,\left[\gamma_{2 r_{2}}, \beta_{2 r_{2}}\right],
$$

yields a shelling order on $\Gamma=\Gamma^{1} \cup \Gamma^{2}$. Namely, the faces in $\left[\alpha_{2 j}, \beta_{2 j}\right] \backslash\left[\gamma_{2 j}, \beta_{2 j}\right]$ are all contained in $\Gamma^{1}$. In particular, the family obtained by removing the intervals 
$\left[\gamma_{2 j}, \beta_{2 j}\right], \ldots,\left[\gamma_{2 r_{2}}, \beta_{2 r_{2}}\right]$ is a pure Boolean cell complex for each $j \in[r]$. By an induction argument, starting with $\Gamma^{1}$, we hence obtain that $\Gamma^{1} \cup \Gamma^{2}$ is shellable.

Proof of Theorem $1.2(i)$. If $P$ is a linear order, then $\Gamma:=\Gamma(\Delta, P)$ is isomorphic to $\Delta$ and hence shellable. Otherwise, let $a \in S$ be maximal such that $a$ is incomparable to some other element in $S$ (with respect to the order on $P$ ). Let $b \in S \backslash a$ be minimal such that $a$ and $b$ are incomparable.

Note that if $c<_{P} b$, then $c<_{P} a$ by minimality of $b$. Analogously, if $a<_{P} c$, then $b<_{P} c$. This implies that if $w$ is a face of $\Gamma$ such that $\{a, b\} \nsubseteq c(w)$, then $w$ is a linear extension of $(P+b a)_{c(w)}$. Namely, by Lemma 2.1, there is a linear extension $w^{\prime}$ of $\left.P\right|_{c(w) \cup\{a, b\}}$ such that $w$ is a subword of $w^{\prime}$. Suppose that $a$ appears before $b$ in $w^{\prime}$. Then all elements $c$ between $a$ and $b$ in $w^{\prime}$ are incomparable to $a$ and $b$ with respect to $P$ by the above properties. In particular, we may insert $a$ just before $b$ or insert $b$ just after $a$ and obtain a word with the desired properties.

As a consequence, we have that

$$
\Gamma(\Delta, P+b a)=\operatorname{fdel}_{\Gamma}(a b) .
$$

In particular, if $a b$ is not in $\Delta$, then $\Gamma$ coincides with $\Gamma(\Delta, P+b a)$. By induction on $P$, we obtain that $\Gamma(\Delta, P)$ is shellable in this case.

If $a b \in \Delta$, then define

$$
\begin{aligned}
& \Gamma^{1}=\Gamma(\Delta, P+b a)=\operatorname{fdel}_{\Gamma}(a b) ; \\
& \Gamma^{2}=\Gamma\left(\mathrm{st}_{\Delta}(a b), P+a b\right)=\mathrm{st}_{\Gamma}(a b) ;
\end{aligned}
$$

the very last equality is a consequence of Lemma 2.2. Note that $\Gamma=\Gamma^{1} \cup \Gamma^{2}$. By induction on $P$, we have that $\Gamma^{1}$ is shellable. Moreover, since

$$
\mathrm{st}_{\Delta}(a b)=2^{\{a, b\}} * \mathrm{lk}_{\Delta}(a b) \cong \operatorname{Cone}^{2}\left(\mathrm{lk}_{\Delta}(a b)\right),
$$

$\mathrm{st}_{\Delta}(a b)$ is shellable; shellability of simplicial complexes is closed under links and cones. Induction on $P$ yields that $\Gamma^{2}$ is shellable.

Using Lemma 3.2, we deduce that $\Gamma=\Gamma^{1} \cup \Gamma^{2}$ is shellable.

Example 3.3. Let $\Delta=\Delta_{M}$ be the simplicial complex of independent sets of a matroid $M$ of rank $n$. Is is well-known that $\Delta_{M}$ is shellable (see [Björner 1992] for this fact and further background on matroids). Thus $\Gamma\left(\Delta_{M}\right)$ is shellable and hence its homology is concentrated in top dimension. As a consequence,

$$
\operatorname{rank}_{\mathbb{Z}} \widetilde{H}_{n-1}\left(\Gamma\left(\Delta_{M}\right) ; \mathbb{Z}\right)=\sum_{F \in \Delta_{M}}(-1)^{n-\# F} \# F ! .
$$

This clearly is a matroid invariant. If $\Delta_{M}$ is the full simplex, then already Farmer's results [1979] show that the left-hand side of (3-1) equals the number of fixed point 
free permutations on \# $M$ letters. We have not been able to recognize the numerical value in (3-1) for other matroids $M$.

Question 3.4. For a given matroid $M$, does there exist a "nice" class of combinatorial objects counted by the value in (3-1)?

A special case of Example 3.3 also appears in [van der Kallen 1980; Suslin 1984, Lemma 2.1]. There the following situation is considered. For finite dimensional vector spaces $V$ and $W$ let $\Delta_{V, W}$ be the simplicial complex of all collections $\left\{\left(v_{0}, w_{0}\right), \ldots,\left(v_{l}, w_{l}\right)\right\}$ of pairs $\left(v_{i}, w_{i}\right) \in V \times W, 0 \leq i \leq l$, such that $v_{0}, \ldots, v_{l}$ are linearly independent. One easily checks that if $V$ and $W$ are vector spaces over a finite field then $\Delta_{V, W}$ indeed is the set of independent sets of a matroid. Now van der Kallen's result [1980], which says that the homology of $\Gamma\left(\Delta_{V, W}\right)$ is concentrated in top dimension, is a special case of Example 3.3.

For the proof of Theorem 1.2 (ii) it will turn out to be profitable to code classes $[w]$ by acyclic orientations. Let $\Delta$ be a simplicial complex on the vertex set $S$ and let $G=(S, E)$ be a simple graph on the same vertex set. To $w \in \Gamma(\Delta)$ we assign a directed graph $D_{w}=\left(c(w), E_{w}\right)$ with vertex set $c(w)$ and with a directed edge from $a$ to $b$ whenever $\{a, b\} \in E$ and $a$ precedes $b$ in the word $w$.

Lemma 3.5. Let $\Delta$ be a simplicial complex on ground set $S$ and let $G=(S, E)$ be a simple graph. Then the following hold:

(i) For $w \in \Gamma(\Delta)$ the directed graph $D_{w}$ is acyclic.

(ii) For $[w] \in \Gamma / G(\Delta)$ and $w^{\prime} \in[w]$ we have $D_{w}=D_{w^{\prime}}$. In particular, the map $[w] \mapsto D_{w}$ is well defined for $[w] \in \Gamma / G(\Delta)$.

(iii) For each face $\sigma \in \Delta$, the map $[w] \mapsto D_{[w]}$ provides a bijection between faces of $\Gamma / G(\Delta)$ with content $\sigma$ and acyclic orientations of the induced sub$\left.\operatorname{graph} G\right|_{\sigma}$.

(iv) The map $[w] \mapsto D_{w}$ is an isomorphism of partially ordered sets between $\Gamma / G(\Delta)$ and the set of acyclic orientations of induced subgraphs $\left.G\right|_{\sigma}$ for $\sigma \in \Delta$ ordered by inclusion of vertex and edge sets.

Proof. (i) Since edges in $D_{w}$ are directed from left to right in $w$, the graph $D_{w}$ cannot have any directed cycles.

(ii) $D_{[w]}$ is well-defined, because if $e=a b \in G$ and $a$ appears before $b$ in some representative $w$, then $a$ appears before $b$ in every representative. This is because any sequence of commutations of neighboring letters transforming $w$ into a word in which $b$ appears before $a$ must contain a step in which $a$ and $b$ are transposed, which is forbidden. 
(iii) To prove that the map is surjective, simply note that every acyclic digraph $D$ on ground set $\sigma$ admits a linear extension $w$, that is, a linear ordering of $\sigma$ such that all edges of $D$ go from smaller to larger vertices. Then clearly $D=D_{w}$.

To prove that the map is injective, suppose that $[w]$ and $\left[w^{\prime}\right]$ yield the same acyclic orientation $D_{w}=D_{w^{\prime}}$. Let $b$ be the first element of $w$ and write $w=b \gamma$ and $w^{\prime}=a_{1} a_{2} \cdots a_{r} b \gamma^{\prime}$, where $\gamma$ and $\gamma^{\prime}$ denote words and $a_{1}, \ldots, a_{r}$ letters. By construction, $D_{w}$ contains no edge directed to $b$. Since $D_{w}=D_{w^{\prime}}$ this implies that $b$ is not adjacent to any $a_{i}, 1 \leq i \leq r$, in $G$. In particular, we may apply a sequence of commutations on $w^{\prime}$ to obtain the word $w^{\prime \prime}=b a_{1} a_{2} \cdots a_{r} \gamma^{\prime}$. By a simple induction argument, we may transform $a_{1} a_{2} \cdots a_{r} \gamma^{\prime}$ into $\gamma$ via a sequence of commutations, which yields that $w$ and $w^{\prime \prime}$, and hence $w$ and $w^{\prime}$, belong to the same commutation class $[w]$.

(iv) By (iii), it remains to verify that $\left[w^{\prime}\right] \preceq[w]$ if and only if $D_{w^{\prime}}$ is the subgraph of $D_{w}$ induced on $c\left(w^{\prime}\right)$. This fact is immediate from the definition of $D_{w}$.

Proof of Theorem 1.2 (ii). By Lemma 3.5, we may identify a given face $[w]$ of $\Gamma$ with the acyclic orientation $D_{w}$ of $\left.G\right|_{c(w)}$ induced by $[w]$.

Fix a linear order on $S$. For vertices $i$ and $j$ of a digraph $D$, we write $i \stackrel{D}{\rightarrow} j$ if there is a directed path from $i$ to $j$. By convention, $i \stackrel{D}{\rightarrow} i$ for all $i$.

In the following we set up functions on vertex sets of digraphs and an order relation on these functions that will later be the key ingredient in the definition of the shelling.

For a digraph $D$ on vertex set $\rho$, define a function $\delta_{D}=\delta: \rho \rightarrow \rho$ by

$$
\delta(i)=\min \{j \mid i \stackrel{D}{\rightarrow} j\} .
$$

Note that $\delta(i) \leq i$ and $\delta^{2}(i)=\delta(i)$ for all $i$. Since $\delta^{2}=\delta$, it is clear that $\delta(i)=i$ whenever $\# \delta^{-1}(\{i\})=1$. For two functions $\delta_{1}, \delta_{2}: \rho \rightarrow \rho$, say that $\delta_{1}>\delta_{2}$ if $\delta_{1}(x) \geq \delta_{2}(x)$ for all $x \in \rho$ with strict inequality for some $x$.

Claim 1. Let $D$ be an acyclic orientation of $\left.G\right|_{\rho}$ and let $x \in \rho$. If $\delta_{D}^{-1}(\{x\})=\{x\}$ then the restriction of $\delta_{D}$ to $\rho \backslash\{x\}$ coincides with $\delta_{D \backslash\{x\}}$.

Proof. Suppose that we have a path from a vertex $y \neq x$ to $x$. By construction, $\delta_{D}(y)=a$ for some $a<x$. Since there is no path from $x$ to $a$ it follows that there is a path from $y$ to $a$ in $D \backslash\{x\}$ and hence that $\delta_{D \backslash\{x\}}(y)=a$.

Claim 2. Let $D$ be an acyclic orientation of $\left.G\right|_{\rho}$ and let $x \in S \backslash \rho$. Then there is a unique acyclic orientation $D^{\prime}$ of $\left.G\right|_{\rho+x}$ containing $D$ such that the restriction of $\delta_{D^{\prime}}$ to $\rho$ coincides with $\delta_{D}$ and such that $\delta_{D^{\prime}}(x)=x$. The digraph $D^{\prime}$ has the property that $\delta_{D^{\prime}}>\delta_{D^{\prime \prime}}$ for all other digraphs $D^{\prime \prime}$ on $\rho+x$ containing $D$.

Proof. Let $A$ be the subset of $\rho$ consisting of all elements $a$ such that $\delta_{D}(a)<x$. Consider an acyclic orientation $D^{\prime \prime}$ of $\left.G\right|_{\rho+x}$ containing $D$. Let $e=x b$ be an edge 
in $\left.G\right|_{\rho+x}$. If $b \in A$ and $e$ is directed from $x$ to $b$, then $\delta_{D^{\prime \prime}}(x) \leq \delta_{D}(b)<x$. If $b \in \rho \backslash A$ and $e$ is directed from $b$ to $x$, then $\delta_{D^{\prime \prime}}(b) \leq x<\delta_{D}(b)$. Thus for the conditions in Claim 2 to hold, we must direct $e$ from $b$ to $x$ whenever $b \in A$ and from $x$ to $b$ whenever $b \in \rho \backslash A$. For the particular face $D^{\prime}$ with this property, one easily checks that the conditions are indeed satisfied. The final statement in Claim 2 follows immediately.

To show that $\Gamma / G(\Delta)$ is shellable, it suffices to verify the following claim.

Claim 3. Let $\tau$ be a maximal face of $\Delta$ and let $\sigma \subseteq \tau$. Then the family

$$
\Gamma_{\sigma, \tau}:=\left\{D_{w} \in \Gamma / G(\Delta) \mid \sigma \subseteq c(w) \subseteq \tau\right\}
$$

admits a partition into intervals

$$
\left[\left.D_{1}\right|_{\sigma_{1}}, D_{1}\right], \ldots,\left[\left.D_{r}\right|_{\sigma_{r}}, D_{r}\right]
$$

such that $\left.D_{i}\right|_{\sigma_{i}}$ is not a subdigraph of $D_{j}$ unless $i \leq j$ and such that each $D_{i}$ is an acyclic orientation of $\left.G\right|_{\tau}$. Here, $\left.D_{i}\right|_{\sigma_{i}}$ is the induced subdigraph of $D_{i}$ on the vertex set $\sigma_{i}$.

Before we proceed to the proof of Claim 3 we provide the arguments that show the sufficiency of Claim 3 for shellability of $\Gamma / G(\Delta)$.

From the shellability of $\Delta$ we deduce from Proposition 3.1 that there is a maximal face $\tau$ and a face $\sigma \subseteq \tau$ such that $\Delta \backslash[\sigma, \tau]$ is shellable. Since $\Gamma / G(\Delta) \backslash \Gamma_{\sigma, \tau}=$ $\Gamma / G(\Delta \backslash[\sigma, \tau])$, Claim 3 implies by inductive applications of Proposition 3.1 that $\Gamma / G(\Delta)$ is shellable.

Proof of Claim 3. Let $D_{1}, \ldots, D_{r}$ be the acyclic orientations of $\left.G\right|_{\tau}$ ordered such that $i<j$ whenever $\delta_{D_{i}}>\delta_{D_{j}}$. For any $D_{i}$, let $X_{i}$ be the set of elements $x \in \tau \backslash \sigma$ such that $\delta_{D_{i}}^{-1}(\{x\})=\{x\}$. Define $\sigma_{i}=\tau \backslash X_{i}$.

We claim that the intervals $\left[\left.D_{i}\right|_{\sigma_{i}}, D_{i}\right]$ yield the desired partition. First, repeated application of (i) yields that $\delta_{\left.D_{i}\right|_{\sigma_{i}}}$ is the restriction of $\delta_{D_{i}}$ to $\sigma_{i}$. Moreover, repeated application of (ii) yields that $\delta_{D_{i}}(x) \geq \delta_{D_{j}}(x)$ for all $x \in \tau$ whenever $\left.D_{i}\right|_{\sigma_{i}}$ is a subdigraph of $D_{j}$ and that the inequality is strict for some $x$, and hence $i<j$, if $D_{i} \neq D_{j}$. By a similar argument, one obtains that any digraph $\left.D_{i}\right|_{\rho}$ such that $\sigma_{i} \subseteq \rho \subseteq \tau$ has the same property. In particular, we obtain the desired claim.

Now, let $[\sigma, \tau]$ be the last interval in the shelling order of $\Delta$. By induction, we know that $\Gamma_{0}=\Gamma / G(\Delta \backslash[\sigma, \tau])$ is shellable. Suppose that we have an ordered partition of the form (3-2) of the remaining family $\{D \in \Gamma / G(\Delta) \backslash \sigma \subseteq V(D) \subseteq \tau\}$ with properties as above. For $1 \leq i \leq r$, define $\Gamma_{i}=\Gamma_{i-1} \cup\left[\left.D_{i}\right|_{\sigma_{i}}, D_{i}\right]$.

We claim that each $\Gamma_{i}$ defines a pure Boolean cell complex; by definition and induction on $i$, this will imply that each $\Gamma_{i}$ is a shellable Boolean cell complex. By assumption the claim is true for $i=0$. Assume that $i>0$. All maximal cells of $\Gamma_{i}$ have the same dimension $\operatorname{dim} \Delta$, because this is true in $\Gamma_{0}$, and $\Gamma_{i}$ is the union of 


\begin{tabular}{|c|c|c|c|c|c|c|c|}
\hline$l$ & $D_{i}$ & $\delta_{D_{i}}$ & $D_{i}\left[\sigma_{i}\right]$ & $i$ & $D_{i}$ & $\delta_{D_{i}}$ & $D_{i}\left[\sigma_{i}\right]$ \\
\hline 1 & 1234 & $12 \underline{3} 4$ * $* *$ & $\varnothing$ & 16 & 2364 & $* 2 \underline{3} 4 * 4$ & 64 \\
\hline 2 & 1243 & 1233 & 43 & 17 & 2436 & $* 233 * \underline{6}$ & 436 \\
\hline 3 & 1423 & $1232 * *$ & 42 & 18 & 2643 & $* 233 * 3$ & 643 \\
\hline 4 & 1342 & $1222 * *$ & 342 & 19 & 4236 & $* 2 \underline{3} 2 * \underline{6}$ & 426 \\
\hline 5 & 3124 & $121 \underline{4}^{* *}$ & 31 & 20 & 6423 & $* 2 \underline{3} 2 * 2$ & 642 \\
\hline 6 & 3142 & 1212 & 3142 & 21 & 3426 & $* 222 * \underline{6}$ & 3426 \\
\hline 7 & 4312 & $1211 * *$ & 431 & 22 & 3642 & $* 222 * 2$ & 3642 \\
\hline 8 & 2134 & $11 \underline{3} \underline{4}^{* *}$ & 21 & 23 & 3456 & $* * 3 \underline{4} 4 \underline{56}$ & 5 \\
\hline 9 & 2143 & $1133^{* *}$ & 2143 & 24 & 3465 & $* * \underline{3} 455$ & 6 \\
\hline 10 & 4213 & $11 \underline{3} 1^{* *}$ & 421 & 25 & 3645 & $* * 3454$ & 645 \\
\hline 11 & 2314 & $111 \underline{4}^{* *}$ & 231 & 26 & 3564 & $* * \underline{3} 444$ & 564 \\
\hline 12 & 2431 & $1111^{* *}$ & 2431 & 27 & 4356 & $* * 33 \underline{56}$ & 435 \\
\hline 13 & 3421 & $1111^{* *}$ & 3421 & 28 & 4365 & $* * 3355$ & 4365 \\
\hline 14 & 4231 & $1111^{* *}$ & 4231 & 29 & 6435 & $* * 33 \underline{5} 3$ & 6435 \\
\hline 15 & 2346 & $* \underline{2} \underline{3} \underline{4} * \underline{6}$ & 6 & 30 & 5643 & $* * 3333$ & 5643 \\
\hline
\end{tabular}

Table 1. Shelling order for the complex $\Gamma / G(\Delta)$, where $\Delta$ and $G$ are defined in Example 3.6.

$\Gamma_{0}$ and a sequence of intervals in which each top element has dimension $\operatorname{dim} \Delta$. It remains to prove that all subfaces of $D_{i}$ belong to $\Gamma_{i}$. Let $\left.D_{i}\right|_{\rho}$ be such a subface. If $\left.D_{i}\right|_{\rho}$ belongs to $\Gamma_{0}$, then we are done. Otherwise, $\left.D_{i}\right|_{\rho} \in\left[\left.D_{j}\right|_{\sigma_{j}}, D_{j}\right]$ for some $1 \leq j \leq r$. By construction, $j \leq i$, which implies that $\left.D_{i}\right|_{\rho} \in \Gamma_{i}$ as desired.

Example 3.6. Let $\Delta$ be the simplicial complex on ground set $S=\{1, \ldots, 5\}$ with maximal faces $1234,2346,3456$. The ordered partition

$$
[\varnothing, 1234],[6,2346],[5,2345]
$$

defines a shelling order of $\Delta$. Let $G$ be the graph with vertex set $S$ and edge set $\{12,13,24,34,46,56\}$. Table 1 provides a shelling order of $\Gamma / G(\Delta)$ constructed as in the proof of Theorem 1.2 (ii) from the given shelling order on $\Delta$ with the natural order on $S$. In the table, each acyclic orientation $D_{i}$ is represented by its lexicographically smallest representative. The function $\delta_{D_{i}}$ is represented as a word $a_{1} a_{2} a_{3} a_{4} a_{5} a_{6}$, where $a_{i}=\delta_{D_{i}}(i)$ if $i \in V\left(D_{i}\right)$ and $a_{i}=*$ otherwise. Underlined values $k$ have the property that $\delta^{-1}(\{k\})=\{k\}$. 
An analysis of the proof of Theorem 1.2 (ii) allows us to describe the rank of the homology groups of $\Gamma / G(\Delta)$ for shellable $\Delta$.

Corollary 3.7. Let $\Delta$ be a shellable d-dimensional simplicial complex on ground set $S$ with shelling order $\left[\sigma_{1}, \tau_{1}\right], \ldots,\left[\sigma_{r}, \tau_{r}\right]$. Fix a linear order $<$ on $S$. Then the rank of the unique nonvanishing reduced homology group $\widetilde{H}_{d}(\Gamma / G(\Delta) ; \mathbb{Z})$ of $\Gamma / G(\Delta)$ equals the number of pairs $\left(\tau_{i}, D\right)$ where $1 \leq i \leq r$ and $D$ is an acyclic orientation of $\left.G\right|_{\tau_{i}}$ such that for all $x \in \tau_{i} \backslash \sigma_{i}$ there is a $y \in \tau_{i} \backslash\{x\}$ such that one of the following conditions holds.

(C1) $y<x$ and there is a directed path from $x$ to $y$ in $D$.

(C2) $y>x$ and there is a directed path from $y$ to $x$ in $D$ and for no $z<x$ there is a directed path from $y$ to $z$ in $D$.

Proof. From the proof of Theorem 1.2 (ii) and Proposition 3.1 we deduce that $\operatorname{rank}_{\mathbb{Z}} \widetilde{H}_{d}(\Gamma / G(\Delta) ; \mathbb{Z})$ is given by the number of pairs $\left(\tau_{i}, D\right)$ where $1 \leq i \leq r$ and $D$ is an acyclic orientation of $\left.G\right|_{\tau_{i}}$ such that for all $x \in \tau_{i} \backslash \sigma_{i}$ we have $\delta_{D}^{-1}(\{x\}) \neq\{x\}$. We distinguish two cases:

(1) $\delta_{D}^{-1}(\{x\})=\varnothing$. In this case there is a $y<x$ for which there is a directed path from $x$ to $y$ in $D$.

(2) $\# \delta_{D}^{-1}(\{x\}) \geq 2$. In this case there is a $y>x$ for which there is a directed path from $y$ to $x$ in $D$ and for no $z<y$ there is a directed path from $y$ to $z$ in $D$.

It is easy to see that (1) and (2) are equivalent to (C1) and (C2), respectively.

Example 3.8. Let $G$ be a graph on the set $S=\{1, \ldots, n\}$ and $\Delta_{n}=2^{S}$ the full simplex. We consider the natural order on $S$. By Corollary 3.7 the rank of the top homology group of $\Gamma / G\left(2^{S}\right)$ is equal to the number of acyclic orientations $D$ of $G$ such that for each vertex $x \in S$ there is a $y \in S$ satisfying at least one of (C1) and (C2) from Corollary 3.7.

(i) For the complete graph $G=K_{n}$, by the work of Farmer [1979] the homology rank is known to be the number of fix-point free permutations. It is an interesting question whether there exists a simple bijection between such permutations and acyclic orientations of $K_{n}$ satisfying the conditions of Corollary 3.7.

(ii) Now we consider the graph $G$ on vertex set $S$ with all edges present except for $\{1,2\},\{2,3\}, \ldots,\{n-1, n\},\{1, n\}$. To avoid trivialities consider only $n \geq 4$. Computer calculations for $4 \leq n \leq 9$ suggest that

$$
\operatorname{rank}_{\mathbb{Z}} \widetilde{H}_{n-1}\left(\Gamma / G\left(\Delta_{n}\right), \mathbb{Z}\right)-(-1)^{n}=a_{n},
$$

where $a_{n}$ is the number of ways to arrange $n$ nonattacking kings on an $n \times n$ chessboard with two sides identified to form a cylinder, with one king in each 
row and one king in each column. Is this indeed true for all $n \geq 4$ ? Is there a nice bijective proof? Note that the left-hand side equals the absolute value of the unreduced Euler characteristic of $\Gamma / G\left(\Delta_{n}\right)$.

We refer the reader to [Abramson and Moser 1967] for more information on the number $a_{n}$. The first few values are $a_{4}=0, a_{5}=10, a_{6}=60, a_{7}=462, a_{8}=3920$, $a_{9}=36954$, and $a_{10}=382740$.

\section{Cohen-Macaulay and sequentially Cohen-Macaulay complexes}

For the formulation of the results of this section we need to review some facts about (sequential) Cohen-Macaulay complexes. Recall that a simplicial complex $\Delta$ is called sequentially homotopy Cohen-Macaulay (SHCM for short) if for all $r \geq 0$ and all $\sigma \in \Delta$ the subcomplex $\left(\mathrm{lk}_{\Delta}(\sigma)\right)^{\langle r\rangle}$ generated by all maximal faces of dimension $\geq r$ in $\operatorname{lk}_{\Delta}(\sigma)$ is $(r-1)$-connected (see for example [Björner et al. 2009]). For $\mathbb{K}$ a field or $\mathbb{K}=\mathbb{Z}$ a simplicial complex $\Delta$ is called sequentially Cohen-Macaulay over $\mathbb{K}(\mathrm{SCM} / \mathbb{K}$ for short $)$ if for all $r \geq 0$ and all $\sigma \in \Delta$ the subcomplex $\left(\mathrm{lk}_{\Delta}(\sigma)\right)^{\langle r\rangle}$ generated by all maximal faces of dimension $\geq r$ in $\operatorname{lk}_{\Delta}(\sigma)$ has vanishing reduced simplicial homology in dimensions 0 through $(r-1)$.

In order to define SHCM, SCM $/ \mathbb{K}, \mathrm{HCM}$ and $\mathrm{CM} / \mathbb{K}$ for partially ordered sets we need to introduce the order complex. For a partially ordered set $Q=\left(M, \leq_{Q}\right)$ on ground set $M$ we denote by $\Delta(Q)=\left\{m_{0}<_{Q} \cdots<m_{l} \mid m_{i} \in M, l \geq-1\right\}$ its order complex. If $Q$ is the face poset of a Boolean cell complex $\Gamma$ then $\Delta(Q)$ is the barycentric subdivision of $\Gamma$.

We call a partially ordered set $Q=\left(M, \leq_{Q}\right)$ on ground set $M$ SHCM (respectively $\mathrm{SCM} / \mathbb{K}, \mathrm{HCM}, \mathrm{CM} / \mathbb{K}$ ) if $\Delta(Q)$ is SHCM (respectively $\mathrm{SCM} / \mathbb{K}, \mathrm{HCM}$, $\mathrm{CM} / \mathbb{K}$ ). In particular, we call a Boolean cell complex $\Gamma$ SHCM (respectively $\mathrm{SCM} / \mathbb{K}, \mathrm{HCM}, \mathrm{CM} / \mathbb{K}$ ) if its barycentric subdivision $\Delta(\Gamma)$ is SHCM (respectively $\mathrm{SCM} / \mathbb{K}, \mathrm{HCM}, \mathrm{CM} / \mathbb{K})$.

A partially ordered set $Q=\left(M, \leq_{Q}\right)$ is called pure if all inclusionwise maximal faces of $\Delta(Q)$ have the same dimension. For $m \in M$, we denote by $Q_{\leq m}$ the subposet of $Q$ on ground set $M_{\leq m}=\left\{m^{\prime} \in M \mid m^{\prime} \leq Q m\right\}$. We call $Q$ semipure if the poset $Q_{\leq m}$ is pure for all $m \in M$. The rank of an element $m \in M$ is the dimension of the simplicial complex $\Delta\left(Q_{\leq m}\right)$. Note that if $Q$ is a pure partially ordered set then the concepts SHCM and HCM (respectively SCM/KK and CM/K ) coincide. It is well-known and easy to prove that shellable Boolean cell complexes are $\mathrm{HCM}$ and hence also $\mathrm{CM} / \mathbb{K}$. For simplicial complexes, it is well-known that the properties of being $\mathrm{SHCM}, \mathrm{SCM} / \mathbb{K}, \mathrm{HCM}$, and $\mathrm{CM} / \mathbb{K}$ are preserved under barycentric subdivision.

The key ingredients to the proof of Theorem 1.3 are Theorem 1.2 and the following results from [Björner et al. 2005]. 
Proposition 4.1 [Björner et al. 2005, Theorem 5.1]. Let $R=\left(N, \leq_{R}\right)$ and $Q=$ $\left(M, \leq_{Q}\right)$ be semipure partially ordered sets and let $f: R \rightarrow Q$ be a surjective and rank-preserving map of partially ordered sets.

(i) Assume that for all $m \in M$ the fiber $\Delta\left(f^{-1}\left(Q_{\leq m}\right)\right)$ is HCM. If $Q$ is SHCM, then so is $R$.

(ii) Let $\mathbb{K}$ be a field or $\mathbb{K}=\mathbb{Z}$. Assume that for all $m \in M$ the fiber $\Delta\left(f^{-1}\left(Q_{\leq m}\right)\right)$ is $\mathrm{SCM} / \mathbb{K}$. If $Q$ is $\mathrm{SCM} / \mathbb{K}$, then so is $R$.

Proof of Theorem 1.3. (i) Consider the map $\phi: \Gamma(\Delta, P) \rightarrow \Delta$ that sends an injective word $\omega_{1} \cdots \omega_{r}$ in $\Gamma(\Delta, P)$ to

$$
\phi\left(\omega_{1} \cdots \omega_{r}\right):=\left\{\omega_{1}, \ldots, \omega_{r}\right\} \in \Delta .
$$

Clearly, $\phi$ is a monotone map if we consider $\Gamma(\Delta, P)$ and $\Delta$ as posets ordered by the subword order and inclusion respectively. Surjectivity is obvious as well. Since the rank of a word from $\Gamma(\Delta, P)$ is given by one less than the cardinality of its content and since the rank of an element of $\Delta$ is again one less than its cardinality the map is rank preserving. Now for a simplex $\sigma \in \Delta$ we study the preimage $\phi^{-1}\left(\Delta_{\leq \sigma}\right)$, which consists of all $\omega_{1} \cdots \omega_{r} \in \Gamma(\Delta, P)$ for which $\left\{\omega_{1}, \ldots, \omega_{r}\right\} \subseteq \sigma$. Hence, if we again denote by $\left.P\right|_{\sigma}$ the restriction of $P$ to $\sigma$ we can identify $\phi^{-1}\left(\Delta_{\leq \sigma}\right)$ with the complex $\Gamma\left(2^{\sigma}, P\right)$. Since the full simplex $2^{\sigma}$ is shellable, $\Gamma\left(2^{\sigma}, P\right)$ is a shellable Boolean cell complex by Theorem 1.2 (i). Therefore, $\Gamma\left(2^{\sigma}, P\right)$ is $\mathrm{HCM}(\mathrm{CM} / \mathbb{K})$. Thus by Proposition 4.1 it follows that $\Gamma(\Delta, P)$ is $\mathrm{SHCM}$ (respectively $\mathrm{SCM} / \mathbb{K}$ ) if $\Delta$ is.

(ii) Consider the map $\phi: \Gamma / G(\Delta) \rightarrow \Delta$ sending a class $\left[\omega_{1} \cdots \omega_{r}\right]$ in $\Gamma(\Delta, P)$ to $\phi\left(\left[\omega_{1} \cdots \omega_{r}\right]\right):=\left\{\omega_{1}, \ldots, \omega_{r}\right\} \in \Delta$. As in the first case, we arrive at the conclusion that $\phi$ is a rank preserving, surjective, and monotone map.

The preimage $\phi^{-1}\left(\Delta_{\leq \sigma}\right)$ of a simplex $\sigma \in \Delta$ consists of all $\left[\omega_{1} \cdots \omega_{r}\right] \in \Gamma / G(\Delta)$ for which $\left\{\omega_{1}, \ldots, \omega_{r}\right\} \subseteq \sigma$. As a consequence, we can identify $\phi^{-1}\left(\Delta_{\leq \sigma}\right)$ with the complex $\Gamma /\left.G\right|_{\sigma}\left(2^{\sigma}\right)$, where $\left.G\right|_{\sigma}$ is the induced subgraph of $G$ on the set $\sigma$. Since $2^{\sigma}$ is shellable, so is $\Gamma /\left.G\right|_{\sigma}\left(2^{\sigma}\right)$ by Theorem 1.2 (ii). Therefore, $\Gamma /\left.G\right|_{\sigma}\left(2^{\sigma}\right)$ is $\operatorname{HCM}(\mathrm{CM} / \mathbb{K})$. Thus by Proposition 4.1 it follows that the poset $\Gamma / G(\Delta)$ is $\mathrm{SHCM}$ (respectively $\mathrm{SCM} / \mathbb{K}$ ) if $\Delta$ is.

\section{Complexes of injective words are partitionable}

A cell complex $\Gamma$ is partitionable if $\Gamma$ admits a partition into pairwise disjoint intervals $\left[\sigma_{i}, \tau_{i}\right]$ such that each $\tau_{i}$ is maximal in $\Gamma$. Any shellable Boolean cell complex is partitionable, but the converse is not true in general. In fact, somewhat surprisingly, for $P$ equal to the antichain $A=\left(S, \leq_{A}\right)$, all complexes of injective words are partitionable: 
Proof of Theorem 1.4. Let $V$ be the vertex set of $\Delta$ and define a total order $\preceq$ on $V$. With the vertices in each face of $\Delta$ arranged in increasing order from left to right, this induces a lexicographic order on the faces. Specifically, let $\sigma \leq \tau$ if and only if either $\tau$ is a prefix of $\sigma$ or if $\sigma$ is lexicographically smaller than $\tau$. For example, for the complex on the vertex set [4] (naturally ordered) with maximal faces 123 and 234 , we have that

$$
123 \prec 12 \prec 13 \prec 1 \prec 234 \prec 23 \prec 24 \prec 2 \prec 34 \prec 3 \prec 4 \prec \varnothing .
$$

For a word $w=w_{0} \cdots w_{r}$, recall that $c(w)=\left\{w_{0}, \ldots, w_{r}\right\}$. Write $\Gamma:=\Gamma(\Delta, A)$. Define a function $f: \Gamma \rightarrow \Delta$ by $f(w)=c(w) \cup f_{0}(w)$, where $f_{0}(w)$ is minimal with respect to $\preceq$ among all faces of $\operatorname{lk}_{\Delta}(c(w))$. Note that $f(w)$ is necessarily a maximal face of $\Delta$. Define another function $g: \Gamma \rightarrow \Gamma$ by letting $g(w)$ be the shortest prefix $v$ of $w$ such that $f(v)=f(w)$. Let $\Gamma^{v}$ be the family of faces $w$ such that $g(w)=v$. It is clear that the families $\Gamma^{v}$ constitute a partition of $\Gamma$.

Now, consider a nonempty family $\Gamma^{v}$. We claim that

$$
\Gamma^{v}=\left\{v w \mid c(w) \subseteq f_{0}(v)\right\} .
$$

Namely, every member of $\Gamma^{v}$ certainly belongs to the set in the right-hand side. Moreover, if $v w$ belongs to this set, then $f_{0}(v)=c(w) \cup f_{0}(v w)$. Namely, suppose that some face $\sigma$ of $\mathrm{lk}_{\Delta}(c(v w))$ is smaller than $f_{0}(v w)$. Then $c(w) \cup \sigma$ is smaller than $c(w) \cup f_{0}(v w)$, which is a contradiction.

As a conclusion, we may write

$$
\Gamma^{v}=v \cdot \Gamma^{\prime}=\left\{v \sigma \mid \sigma \in \Gamma^{\prime}\right\},
$$

where $\Gamma^{\prime}$ is the complex of injective words derived from the full simplex on the vertex set $f_{0}(v)$. Since $\Gamma^{\prime}$ is shellable, we may partition $\Gamma^{\prime}$ into intervals $[u, w]$ such that each top cell $w$ is maximal in $\Gamma^{\prime}$. This induces a partition of $\Gamma^{v}$ into intervals $[v u, v w]$ such that each top cell $v w$ is maximal in $\Gamma^{v}$ and hence in $\Gamma$.

The $h$-polynomial $h(\Gamma ; t):=\sum_{i} h_{i} t^{i}$ of a Boolean cell complex of dimension $d$ is defined by

$$
\sum_{i} h_{i} t^{i}=\sum_{i} f_{i} t^{i}(1-t)^{d+1-i} \Longleftrightarrow \sum_{i} f_{i} t^{i}=\sum_{i} h_{i} t^{i}(1+t)^{d+1-i},
$$

where $f_{i}$ is the number of cells of dimension $i-1$ in $\Gamma$.

Corollary 5.1. Let $\Delta$ be a pure simplicial complex. Then all coefficients of the h-polynomial of $\Gamma(\Delta)$ are nonnegative.

Proof. By Theorem 1.4, we may partition $\Gamma(\Delta)$ into a disjoint union of intervals $\left[\sigma_{i}, \tau_{i}\right]$ such that each $\tau_{i}$ has maximum dimension $d$. It follows that $h(\Gamma(\Delta) ; t)=$ $\sum t^{\# \sigma_{i}}$. 
A partition into intervals of a Boolean cell complex $\Gamma$ induces a matching of cells such that the only unmatched cells in the complex are the ones that form singleton intervals $[\tau, \tau]$ in the partition. Specifically, consider the graph on the set of faces of $\Gamma$, where we have an edge between two faces $\sigma$ and $\tau$ whenever $\sigma<\tau$ and there is no face $\gamma$ for which $\sigma<\gamma<\tau$. Thus this graph is the graph of the Hasse diagram of $\Gamma$. Now, for two faces $\sigma<\tau$ of $\Gamma$, each interval $[\sigma, \tau]$ is a Boolean lattice and therefore the associated Hasse diagram has a perfect matching if and only if $\sigma \neq \tau$. In particular, this shows that on the Hasse diagram of a partitionable Boolean cell complex there is a matching whose only unmatched faces are the ones corresponding to one-element intervals. In discrete Morse theory [Forman 1998], matchings of the Hasse diagram of the face poset of a regular CW-complex are used to determine the topological structure of the complex. However, in general, discrete Morse theory [Forman 1998] does not apply to the matchings constructed above. Namely, a matching relevant to discrete Morse theory has to satisfy the additional assumption that if one directs all edges from the matching upward by dimension and all other edges downward, then the resulting directed graph must be acyclic.

For example, consider the complex with maximal faces 12 and 34. The induced order of the faces is

$$
12 \prec 1 \prec 2 \prec 34 \prec 3 \prec 4 \prec \varnothing,
$$

which yields $\Gamma^{\varnothing}=\{\varnothing, 1,2,12,21\}, \Gamma^{3}=\{3,34\}$ and $\Gamma^{4}=\{4,43\}$. We obtain a partition consisting of the four intervals [Ø, 12], [21, 21], [3, 34] and [4, 43], which yields a matching including the pairs $\{3,34\}$ and $\{4,43\}$. This is illegal in terms of discrete Morse theory.

\section{References}

[Abramson and Moser 1967] M. Abramson and W. O. J. Moser, "Permutations without rising or falling $w$-sequences", Ann. Math. Statist. 38 (1967), 1245-1254. MR 35 \#2752 Zbl 0153.01903

[Björner 1992] A. Björner, "The homology and shellability of matroids and geometric lattices", pp. 226-283 in Matroid applications, edited by N. White, Encyclopedia Math. Appl. 40, Cambridge Univ. Press, 1992. MR 94a:52030 Zbl 0772.05027

[Björner 1995] A. Björner, "Topological methods", pp. 1819-1872 in Handbook of combinatorics, Vol. 2, edited by R. L. Graham et al., Elsevier, Amsterdam, 1995. MR 96m:52012 Zbl 0851.52016

[Björner and Wachs 1983] A. Björner and M. L. Wachs, "On lexicographically shellable posets", Trans. Amer. Math. Soc. 277:1 (1983), 323-341. MR 84f:06004 Zbl 0514.05009

[Björner et al. 2005] A. Björner, M. L. Wachs, and V. Welker, "Poset fiber theorems", Trans. Amer. Math. Soc. 357:5 (2005), 1877-1899. MR 2006a:05172 Zbl 1086.55003

[Björner et al. 2009] A. Björner, M. L. Wachs, and V. Welker, "On sequentially Cohen-Macaulay complexes and posets”, Israel J. Math. 169 (2009), 295-316. MR 2460907 Zbl 05508742

[Farmer 1979] F. D. Farmer, “Cellular homology for posets”, Math. Japon. 23:6 (1979), 607-613. MR 82k:18013 Zbl 0416.55003 
[Forman 1998] R. Forman, "Morse theory for cell complexes", Adv. Math. 134:1 (1998), 90-145. MR 99b:57050 Zbl 0896.57023

[Gerdes 1991] W. Gerdes, "Affine Grassmannian homology and the homology of general linear groups", Duke Math. J. 62:1 (1991), 85-103. MR 92i:14006 Zbl 0743.14007

[Hanlon and Hersh 2004] P. Hanlon and P. Hersh, "A Hodge decomposition for the complex of injective words", Pacific J. Math. 214:1 (2004), 109-125. MR 2005c:13019 Zbl 1086.16005

[van der Kallen 1980] W. van der Kallen, "Homology stability for linear groups", Invent. Math. 60:3 (1980), 269-295. MR 82c:18011 Zbl 0415.18012

[Kerz 2005] M. C. Kerz, "The complex of words and Nakaoka stability", Homology Homotopy Appl. 7:1 (2005), 77-85. MR 2006d:18005 Zbl 1090.18009

[Knudson 2001] K. P. Knudson, Homology of linear groups, Progress in Math. 193, Birkhäuser Verlag, Basel, 2001. MR 2001j:20070 Zbl 0997.20045

[Mirzaii and van der Kallen 2002] B. Mirzaii and W. van der Kallen, "Homology stability for unitary groups”, Doc. Math. 7 (2002), 143-166. MR 2003e:19007 Zbl 0999.19005

[Ragnarsson and Tenner 2009] K. Ragnarsson and B. E. Tenner, "Homotopy type of the Boolean complex of a Coxeter system", Adv. Math. 222:2 (2009), 409-430. arXiv 0806.0906

[Reiner and Webb 2004] V. Reiner and P. Webb, "The combinatorics of the bar resolution in group cohomology”, J. Pure Appl. Algebra 190:1-3 (2004), 291-327. MR 2005i:57001 Zbl 1057.57003

[Suslin 1984] A. A. Suslin, "Homology of $\mathrm{GL}_{n}$, characteristic classes and Milnor $K$-theory", pp. 357-375 in Algebraic K-theory, number theory, geometry and analysis (Bielefeld, 1982), edited by A. Bak, Lecture Notes in Math. 1046, Springer, Berlin, 1984. MR 86f:11090a Zbl 0528.18007

Received February 26, 2008. Revised May 17, 2009.

\author{
JAKOB JONSSON \\ DEPARTMENT OF MATHEMATICS \\ KTH \\ 10044 STOCKHOLM \\ SWEDEN \\ jakobj@math.kth.se \\ http://www.math.kth.se/ jakobj/
}

VOLKMAR WELKER

FACHBEREICH MATHEMATIK UND INFORMATIK

PHILIPPS-UNIVERSITÄT MARBURG

35032 MARBURG

GERMANY

welker@mathematik.uni-marburg.de

www.mathematik.uni-marburg.de/ welker 